\author{
A.Kh. Attaev \\ Institute of Applied Mathematics and Automation of Kabardino-Balkarian \\ Scientific Center, Russian Academy of Sciences, Nalchik, Russia \\ (E-mail: attaev.anatoly@yandex.ru)
}

\title{
On a characteristic problem for a loaded hyperbolic equation
}

\begin{abstract}
The paper studies a loaded hyperbolic equation with one-dimensional wave equation in its main part. In the loaded components there are two points, which are distributed respectively along a pair of intersecting characteristics at a constant speed. For such an equation we study the Cauchy problem with characteristics of the one dimensional wave equation belonging to any of the pair of intersecting straight lines. If to impose certain conditions at the boundary points on function presenting Cauchy data and the derivatives of the first, second and third orders we can prove the existence and uniqueness of the problem. The proof of the existence and uniqueness of the solution follows directly from the method of its production. We consider also issues concerning domains of dependence, influence, and definitions for Cauchy data that are specified on one of the characteristic curves. The above verifies once more the thesis of load influencing on posing of various initial - boundary value problem for partial differential equations.
\end{abstract}

Keywords: Cauchy problem, loaded equation, wave equation, characteristics, domain of influence, domain of dependence.

\section{Introduction}

A.M. Nakhushev first made the most general definition for a loaded equation in [1]. In [2] he had introduced concepts and a detailed classification for various loaded equations: loaded differential, loaded integral, loaded integrodifferential, loaded functional equations. Besides fundamental work on the study of loaded integral and loaded ordinary differential equations, we wish to acknowledge some of the proceedings [3-6]. Together with A.M. Nakhushev and his successors [7-16] a systematic researches and significant contribution to the boundary value problems for loaded differential equations had been made by the Kazakh mathematicians M.T. Dzhenaliev and M.I.Ramazanov, and their students $[17,18]$. They investigated a wide class of homogeneous and inhomogeneous boundary value problems for essentially loaded parabolic and hyperbolic-elliptic equations, as well as the spectral issues on the corresponding homogeneous problems, when the load is specified with respect to the space variable and the load point moves at constant and variable speeds. Works [19-21] are devoted to uniqueness classes for solution of the Cauchy problem and non-trivial solutions of the homogeneous Cauchy problem for some classes of loaded differential equations and linear loaded systems of the first order.

The Cauchy problem and the Cauchy-Dirichlet problem for a spectrally loaded parabolic equation with a load at a fixed time variable are investigated in $[22,23]$.

The effect of the load on the convergence of spectral expansions for operators is considered in [24].

In [25-27] the Goursat problem for second-order strictly and weakly hyperbolic equations with two independent variables is investigated, where it is shown that the load allows eliminating the inequality between characteristics that are data in the Goursat problem.

This paper considers the following loaded equation

$$
u_{x x}-u_{y y}=\lambda u(x+y, 0)+\mu u(x-y, 0)
$$

where $\lambda, \mu$ are arbitrary real constants.

Using characteristic variables $\xi=x-y, \quad \eta=x+y$ equation (1) has the form

$$
v_{\xi \eta}=\frac{\lambda}{4} v(\eta, \eta)+\frac{\mu}{4} v(\xi, \xi)
$$

where $v(\xi, \eta)=u\left(\frac{\xi+\eta}{2}, \frac{\eta-\xi}{2}\right)$. 
By (2) it follows that

$$
v(\xi, \eta)=f(\xi)+g(\eta)+\frac{\lambda}{4} \xi \int_{0}^{\eta} v(t, t) d t+\frac{\mu}{4} \eta \int_{0}^{\xi} v(t, t) d t,
$$

where $f(\xi), g(\eta)$ are arbitrary smooth enough functions.

As $\eta=\xi$ by (3) obtain

$$
\frac{d}{d \xi}\left[e^{-\frac{\lambda+\mu}{8} \xi^{2}} \int_{0}^{\xi} v(t, t) d t\right]=e^{-\frac{\lambda+\mu}{8} \xi^{2}}[f(\xi)+g(\xi)] .
$$

Hence

$$
\int_{0}^{\xi} v(t, t) d t=\int_{0}^{\xi} e^{\frac{\lambda+\mu}{8}\left(\xi^{2}-t^{2}\right)}[f(t)+g(t)] d t .
$$

Substituting into (3) instead of integrals values obtained using formula (4) and moving to $x, y$, coordinates we get

$$
\begin{gathered}
u(x, y)=f(x-y)+g(x+y)+\frac{\lambda}{4}(x-y) \int_{0}^{x+y} e^{\frac{\lambda+\mu}{8}\left[(x+y)^{2}-t^{2}\right]}[f(t)+g(t)] d t+ \\
+\frac{\mu}{4}(x+y) \int_{0}^{x-y} e^{\frac{\lambda+\mu}{8}\left[(x-y)^{2}-t^{2}\right]}[f(t)+g(t)] d t .
\end{gathered}
$$

Formula (5) is an analogue of d'Alembert formula for equation (1) and obviously as $\lambda=\mu=0$ coincides with the d'Alembert formula for equation (1) as $\lambda=\mu=0$.

Assume $\Omega=\{(x, y): 0<x+y<1,0<x-y<1\}$ is a characteristic quadrilateral.

Cauchy problem. In the domain $\bar{\Omega}$ find a regular solution $\mathrm{u}(\mathrm{x}, \mathrm{y})$ of equation (1) continuous in $\bar{\Omega}$ and satisfying the conditions

$$
\begin{gathered}
u\left(\frac{x}{2}, \frac{x}{2}\right)=\varphi(x), \quad 0 \leq x \leq 1, \\
u_{y}\left(\frac{x}{2}, \frac{x}{2}\right)=\psi(x), \quad 0 \leq x \leq 1,
\end{gathered}
$$

where $\varphi(x), \psi(x)$ - are specified functions.

It is well known that as $\lambda=\mu=0$ this problem is not well posed. The necessary and sufficient condition for its solvability is

$$
\varphi^{\prime}(x)-\psi(x)=\varphi^{\prime}(0)-\psi(0), \quad 0 \leq x \leq 1 .
$$

If the above condition is satisfied, the function $u(x, y)$ is a solution to problem $(6),(7)$ for equation (1) and has the form

$$
u(x, y)=f(x-y)-f(0)+\varphi(x+y),
$$

where $f$ is an arbitrary twice continuously differentiable function.

The following theorem is valid.

Theorem. Assume $\lambda \neq 0, \varphi \in C^{3}(\bar{J}) \cap C^{4}(J), \psi \in C^{2}(\bar{J}) \cap C^{3}(J)$ and the coordination conditions

$$
\begin{gathered}
\varphi(0)=\psi(0)=\varphi^{\prime}(0)=0, \\
\varphi^{\prime \prime}(0)-\psi^{\prime}(0)=0, \\
\varphi^{\prime \prime \prime}(0)-\psi^{\prime \prime}(0)=0 .
\end{gathered}
$$

Then the solution for the Cauchy problem exists, is unique and representable in the form of

$$
\begin{gathered}
u(x, y)=\frac{4}{\lambda}\left[\varphi^{\prime \prime}(x-y)-\psi^{\prime}(x-y)\right]-\left(x-\frac{\lambda+2 \mu}{\lambda} y\right)\left[\varphi^{\prime}(x-y)-\psi(x-y)\right]+ \\
+(x-y)\left[\varphi^{\prime}(x+y)-\psi(x+y)\right]+\varphi(x+y)-\varphi(x-y) .
\end{gathered}
$$

Indeed the function $u(x, y)$ is a solution to the Cauchy problem if and only if it is representable as (5). Therefore, by substituting (5) into (6) and (7), we can get

$$
\begin{gathered}
f(0)+g(x)=\varphi(x) \\
-f^{\prime}(0)+g^{\prime}(x)-\frac{\lambda}{4} \int_{0}^{x} e^{\frac{\lambda+\mu}{8}\left(x^{2}-t^{2}\right)}[f(t)+g(t)] d t-
\end{gathered}
$$




$$
-\frac{\mu}{4} x[f(0)+g(0)]=\psi(x)
$$

Taking into account (8) from (12) and (13) it is clear that

$$
f(0)+g(0)=0, \quad f^{\prime}(0)=0 .
$$

Hence, by (12) and (13) we have

$$
\begin{gathered}
g(x)=\varphi(x)-f(0) \\
\int_{0}^{x} e^{\frac{\lambda+\mu}{8}\left(x^{2}-t^{2}\right)}[f(t)+g(t)] d t=\frac{4}{\lambda}\left[\varphi^{\prime}(x)-\psi(x)\right] .
\end{gathered}
$$

By differentiation of (15) with respect to $x$ and subtracting identity (15), previously multiplied by $\frac{\lambda+\mu}{4} x$ we can get

or

$$
f(x)+g(x)=\frac{4}{\lambda}\left[\varphi^{\prime \prime}(x)-\psi^{\prime}(x)\right]-\frac{\lambda+\mu}{\lambda} x\left[\varphi^{\prime}(x)-\psi(x)\right]
$$

$$
f(x)=f(0)-\varphi(x)+\frac{4}{\lambda}\left[\varphi^{\prime \prime}(x)-\psi^{\prime}(x)\right]-\frac{\lambda+\mu}{\lambda} x\left[\varphi^{\prime}(x)-\psi(x)\right] .
$$

Substituting the obtained values into $f(x)$ and $g(x)$ in (5) and taking into account (15) when calculating the last two terms of formula (5), and after simple conversions we arrive at formula (11).

Substitution into equation (1) ensures us that the function $u(x, y)$ calculated by formula (11) is the solution to (1). It is easy to check that under conditions (8)-(10) the function $u(x, y)$ satisfies conditions (6), (7).

Note that the Cauchy problem as $\lambda=0$ is not a well posed one. Indeed by (12), (13) the condition

$$
\varphi^{\prime}(x)-\psi(x)=\varphi^{\prime}(0)-\psi(0)+\frac{\mu}{4} \varphi(0)
$$

is necessary and sufficient for the solvability of the problem. In case this condition is satisfied the solution to the Cauchy problem is

$$
u(x, y)=f(x-y)-f(0)+\varphi(x+y)+\frac{\mu}{4}(x+y) \int_{0}^{x-y}[f(t)-f(0)+\varphi(t)] e^{\frac{\mu}{4}\left[(x-y)^{2}-t^{2}\right]} d t
$$

where $f-$ is an arbitrary twice continuously differentiable function.

If we make a replacement $u(x, y)=v(x,-y)$ in equation (1) then problem

$$
\begin{aligned}
& u\left(\frac{x}{2},-\frac{x}{2}\right)=\varphi(x), \quad 0 \leq x \leq 1, \\
& u_{y}\left(\frac{x}{2},-\frac{x}{2}\right)=\psi(x) \quad 0 \leq x \leq 1,
\end{aligned}
$$

becomes the problem

$$
v\left(\frac{x}{2}, \frac{x}{2}\right)=\varphi(x), \quad v_{y}\left(\frac{x}{2}, \frac{x}{2}\right)=-\psi(x)
$$

for the equation $v_{x x}-v_{y y}=\mu v(x+y, 0)+\lambda v(x-y, 0)$.

Therefore, assuming that $\mu \neq 0$ and

$$
\varphi(0)=\psi(0)=\varphi^{\prime}(0)=0 ; \quad \varphi^{\prime \prime}(0)+\psi^{\prime}(0)=0 ; \quad \varphi^{\prime \prime \prime}(0)+\psi^{\prime \prime}(0)=0,
$$

the solution to the problem is representable as

$$
\begin{gathered}
v(x, y)=\frac{\mu}{4}\left[\varphi^{\prime \prime}(x-y)+\psi^{\prime}(x-y)\right]-\left(x-\frac{\mu+2 \lambda}{\mu} y\right)\left[\varphi^{\prime}(x-y)+\psi(x-y)\right]+ \\
+(x-y)\left[\varphi^{\prime}(x+y)+\psi(x+y)\right]+\varphi(x+y)-\varphi(x-y) .
\end{gathered}
$$

This implies the solution to problem (16), (17) for equation (1) casts into the form

$$
\begin{gathered}
u(x, y)=\frac{\mu}{4}\left[\varphi^{\prime \prime}(x+y)+\psi^{\prime}(x+y)\right]-\left(x+\frac{\mu+2 \lambda}{\mu} y\right)\left[\varphi^{\prime}(x+y)+\psi(x+y)\right]+ \\
+(x+y)\left[\varphi^{\prime}(x-y)+\psi(x-y)\right]+\varphi(x-y)-\varphi(x+y) .
\end{gathered}
$$


It is known [28] that in case with three spatial variables corresponding to the Cauchy problem a wave is completely determined by the Cauchy data on a sphere. This fact in the theory of sound is called Huygens principle. We also know that with two spatial variables in wave processes the Huygens principle does not hold since to determine the wave the Cauchy data must be specified not only on the circle but also at all points of the corresponding circle. In the case of one variable, to determine the value of the oscillation at the point $(x, y)$, one of the components of the Cauchy data must be set on the segment boundary $[x-y, x+y]$, and the second at all points of this segment.

The idea is that to determine $u(x, y)$ at the point $(x, y)$ in formulas (11), (18) you need to know the Cauchy data only on the segment boundary $[x-y, x+y]$. That is, we can say that there is a one-dimensional version for the Huygens principle.

\section{References}

1 Нахушев А.М. О задаче Дарбу для одного вырождающегося нагруженного интегро-дифференциального уравнения второго порядка / А.М. Нахушев // Дифференциальные уравнения. - 1976. - T. 12, № 1. - C. 103-108.

2 Нахушев А.М. Уравнения математической биологии / А.М. Нахушев. - М.: Высш. шк., 1995. - 301 с.

3 Knezer A. Rendiconti del Cirolo Matematico di Palermo / A. Knezer. - 1914. - V. 37. - P. 169-197.

4 Крейн М.Г. О нагруженных интегральных уравнениях, функции распределения которых не монотонны / Д.А. Траве // Сб. памяти акад. - М.; Л., 1940. - С. 88-103.

5 Krall A.M. Differential - boundary operators: Trans. Amer. Math. Soc. - 1971. - 154 p.

6 Phillips R.S. Dissipativ operators and hyperbolic systems of partional differential equations / R.S. Phillips // Tpons. Amer. Math. Sos. - 1990. - P. 193-254.

7 Нахушев А.М. Нагруженные уравнения и их приложения / А.М. Нахушев. - М.: Наука, 2012. - 232 с.

8 Нахушев А.М. О нелокальных краевых задачах со смещением и их связи с нагруженными уравнениями / А.М. Нахушев // Дифференциальные уравнения. - 1985. - Т. 21, № 1. - С. 92-101.

9 Нахушев А.М. Нелокальные задачи и задача Гурса для нагруженного уравнения гиперболического типа и их приложения к прогнозу почвенной влаги / А.М. Нахушев // Докл. АН СССР. - 1978. T. 242, № 5. - C. 1008-1011.

10 Нахушев А.М. Краевые задачи для нагруженных интегро-дифференциальных уравнений гиперболического типа и некоторых их приложениях к прогнозу почвенной влаги / А.М. Нахушев // Дифференциальные уравнения. - 1979. - Т. 15, № 1. - С. 96-105.

11 Нахушева В.А. Дифференциальные уравнения математических моделей нелокальных процессов / А.М. Нахушев. - М.: Наука, 2006. - 174 с.

12 Елеев В.А. О некоторых краевых задачах для смешанных нагруженных уравнений второго и третьего порядка / В.А. Елеев // Дифференциальные уравнения. - 1994. - Т. 30, № 2. - С. 230-237.

13 Псху А.В. Уравнения в частных производных дробного порядка / А.В. Псху. - М.: Наука, 2005. - 199 с.

14 Казиев В.М. Задача Гурса для одного нагруженного интегро-дифференциального уравнения / В.М. Казиев // Дифференциальные уравнения. - 1981. - Т. 17, № 2. - С. 313-319.

15 Хубиев К.У. Аналог задачи Трикоми для характеристически нагруженного уравнения гиперболопараболического типа с переменными коэффициентами / К.У. Хубиев // Уфим. математ. журн. 2017. - T. 9, № 2. - С. 94-103.

16 Хубиев К.У. Задачи со смещением для нагруженного уравнения гиперболо-параболического типа с оператором дробной диффузии / К.У. Хубиев // Вестн. Удмурт. ун-та. Сер. Математика. Механика. Компьютерные науки. - 2018. - Т. 28, № 1. - С. 82-90.

17 Дженалиев М.Т. К теории линейных краевых задач для нагруженных дифференциальных уравнений / М.Т. Дженалиев. - Алматы: Компьютерный центр ТИПМ, 1995. - 270 с.

18 Дженалиев М.Т. Нагруженные уравнения как возмущения дифференциальных уравнений / М.Т. Дженалиев, М.И. Рамазанов. - Алматы: Ғылым, 2010. - 334 с.

19 Огородников Е.Н. Некоторые характеристические задачи для систем нагруженных дифференциальных уравнений и их связь с нелокальными краевыми задачами / Е.Н. Огородников // Вестн. Самар. гос. техн. ун-та. Сер. Физ.-мат. науки. - 2003. - Т. 19. - С. 22-28. 
20 Борок В.М. О существовании и единственности решения задачи Коши для нагруженных уравнений / В.М. Борок // Изв. вузов. Сер. Матем. - 1986. - № 4. - С. 8-13.

21 Борок В.М. О единственности решения задачи Коши для систем линейных нагруженных уравнений / В.М. Борок // Украин. математ. журн. - 1985. - Т. 37, № 1. - С. 108-109.

22 Дженалиев М.Т. О задаче Коши для существенно нагруженного параболического уравнения / М.Т. Дженалиев, М.И. Рамазанов // Математ. журн. - 2004. - Т. 4, № 3(13). - С. 22-26.

23 Дженалиев М.Т. О гранично-начальной задаче для существенно нагруженного параболического уравнения / М.Т. Дженалиев, М.И. Рамазанов // Изв. НАН РК, Сер. физ-мат. - 2005. - № 5. C. $36-43$.

24 Ломов И.С. Нагруженные дифференциальные операторы: сходимость спектральных разложений / И.С. Ломов // Дифференциальные уравнения. - 1983. - Т. 19, №1. - С. 86-94.

25 Аттаев А.Х. Задача Гурса для нагруженного вырождающегося гиперболического уравнения второго порядка с оператором Геллерстедта в главной части / А.Х. Аттаев // Вестн. Самар. гос. техн. ун-та. Сер. Физ.-мат. науки. - 2016. - №1 (20). - С. 7-22.

26 Аттаев А.Х. Задача Гурса для нагруженного гиперболического уравнения / А.Х. Аттаев // Докл. Адыгской (Черкесской) МАН. - 2014. - Т. 16, № 3. - С. 9-12.

27 Аттаев А.Х. Задача с данными на параллельных характеристиках для нагруженного волнового уравнения / А.Х. Аттаев // Докл. Адыгской (Черкесской) МАН. - 2013. - Т. 15. - №2. - С. 25-28.

28 Бицадзе А.В. Уравнения математической физики / А.В. Бицадзе. - М.: Наука, 1982. - 336 с.

\section{А.Х. Аттаев \\ Жүктелген гиперболалық теңдеу үшін бір сипаттамалық есеп жайлы}

Бұл жұмыста басты бөлігі шектің тербелісінің бірөлшемді теңдеуі болатын жүктелген гиперболалық теңдеу объект болып табылады. Жүктелген қосылғыштарда тұрақты жылдамдықпен қиылысатын сипаттаушы жұптарының бойына сәйкес таралатын жүктеменің екі нүктесі бар. Осындай теңдеу үшін бірөлшемді шектің тербелісінің теңдеуінің сипаттаушылары болып табылатын қиылысатын түзулердің кез келген жұбының деректерімен Коши есебін зерттеу жүргізілді. Коши деректерін беретін функцияға нүктелік сипаттағы белгілі шарттарда және олардың бірінші, екінші, үшінші туындылары қойылған есептің бар болуы және жалғыздығы дәләлденді, ал шешуі жайлы түсінік анық жазылды. Шешудің бар болуы және жалғыздығын дәлелдеу оны алу әдісінің өзінен шығады. Сонымен қоса бір сипаттаушыда берілетін, Коши деректерінің тәуелділік, әсер ету және анықталу облыстарымен байланысты сұрақтар қарастырылды. Осылайша дербес туындылы жүктемелі дифференциалдық теңдеулер үшін бастапқы-шектік есептердің қойылымына жүктеменің әсерінің тиімділігі жайлы тезис кезекті рет нақтыланды.

Kiлm сөздер: Коши есебі, жүктемелі теңдеу, шектің тербеліс теңдеуі, сипаттамалар, әсер ету облысы, тәуелсіздік облысы.

\section{А.Х. Аттаев \\ Об одной характеристической задаче для нагруженного гиперболического уравнения}

\footnotetext{
Объектом исследования статьи является нагруженное гиперболическое уравнение, главная часть которого представляет собой одномерное уравнение колебания струны. В нагруженных слагаемых присутствуют две точки нагрузки, которые распространяются соответственно вдоль пары пересекающихся характеристик с постоянной скоростью. Для такого уравнения проводится исследование задачи Коши с данными на любой из пары пересекающихся прямых, являющихся характеристиками уравнения колебания одномерной струны. При определенных условиях точечного характера на функции,
} 
задающие данные Коши и производные первого, второго и третьего порядков от них, доказывается существование и единственность поставленной задачи, а представление самого решения выписывается в явном виде. Доказательство существования и единственности решения непосредственно следует из самого способа его получения. Также затрагиваются вопросы, связанные с областями зависимости, влияния и определения данных Коши, задаваемых на одной из характеристик. Этим самым в очередной раз подтверждается тезис об эффекте влияния нагрузки на постановку тех или иных начально-краевых задач для нагруженных дифференциальных уравнений с частными производными.

Ключевые слова: задача Коши, нагруженное уравнение, уравнение колебания струны, характеристики, область влияния, область зависимости.

\section{References}

1 Nakhushev, A.M. (1976). O zadache Darbu dlia odnoho vyrozhdaiushchehosia nahruzhennoho intehrodifferentsialnoho uravneniia vtoroho poriadka [On the Darboux problem for a single degenerate loaded second-order integro-differential equation]. Differentsialnye uravneniia - Differential Equations, Vol. 12, No. 1, 103-108 [in Russian].

2 Nakhushev, A.M. (1995). Uravneniia matematicheskoi biolohii [Equations of mathematical biology]. Moscow: Vysshaia shkola [in Russian].

3 Knezer, A. (1914). Rendiconti del Cirolo Matematico di Palermo, Vol. 37, 169-197.

4 Kerin, M.G. (1940). O nahruzhennykh intehralnykh uravneniiakh, funktsii raspredeleniia kotorykh ne monotonny [On loaded integral equations with non-monotonous distribution functions]. Sb. Memory Acad. D.A. Trave. Moscow; Leningrad, 88-103 [in Russian].

5 Krall, A.M. (1971). Differential - boundary operators: Trans. Amer. Math. Soc.

6 Phillips, R.S. (1990). Dissipativ operators and hyperbolic systems of partional differential equations. Tpons. Amer. Math. Sos., 193-254.

7 Nakhushev, A.M. (2012). Nahruzhennye uravneniia $i$ ikh prilozheniia /Loaded equations and their applications]. Moscow: Nauka [in Russian].

8 Nakhushev, A.M. (1985). O nelokalnykh kraevykh zadachakh so smeshcheniem i ikh sviazi s nahruzhennymi uravneniiami [On nonlocal boundary value problems with displacement and their relationship with loaded equations]. Differentsialnye uravneniia - Differential Equations, Vol. 21, no. 1, 92-101 [in Russian].

9 Nakhushev, A.M. (1978). Nelokalnye zadachi i zadacha Hursa dlia nahruzhennoho uravneniia hiperbolicheskoho tipa i ikh prilozheniia k prohnozu pochvennoi vlahi [Nonlocal problems and the Goursat problem for a loaded hyperbolic type equation and their application to the soil moisture forecast]. Doklady AN SSSR - Doklady Academy of Sciences of the USSR, Vol. 242, No. 5, 1008-1011 [in Russian].

10 Nakhushev, A.M. (1979). Kraevye zadachi dlia nahruzhennykh intehro-differentsialnykh uravnenii hiperbolicheskoho tipa i nekotorye ikh prilozheniiakh $\mathrm{k}$ prohnozu pochvennoi vlahi [Boundary value problems for loaded integro-differential equations of hyperbolic type and some of their applications to the soil moisture prediction]. Differentsialnye uravneniia - Differential Equations, Vol. 15, No. 1, 96-105 [in Russian].

11 Nakhusheva, V.A. (2006). Differentsialnye uravneniia matematicheskikh modelei nelokalnykh protsessov [Differential equations of mathematical models of non-local processes]. Moscow: Nauka [in Russian].

12 Eleev, V.A. (1994). O nekotorykh kraevykh zadachakh dlia smeshannykh nahruzhennykh uravnenii vtoroho i treteho poriadka [On some boundary value problems for mixed loaded equations of the second and third orders]. Differentsialnye uravneniia - Differential Equations, Vol. 30, No. 2, 230-237 [in Russian].

13 Psku, A.V. (2005). Uravneniia v chastnykh proizvodnykh drobnoho poriadka [Differential equations with partial derivatives]. Moscow: Nauka [in Russian].

14 Kaziev, V.M. (1981). Zadacha Hursa dlia odnoho nahruzhennoho intehro-differentsialnoho uravneniia [The Goursat problem for a single loaded integro-differential equation]. Differentsialnye uravneniia Differential Equations, Vol. 17, No. 2, 313-319 [in Russian].

15 Khubiev, K.U. (2017). Analoh zadachi Trikomi dlia kharakteristicheski nahruzhennoho uravneniia hiperbolo-parabolicheskoho tipa s peremennymi koeffitsientami [Analog of the Tricomi problem for a characteristic loaded hyperbolic-parabolic equation with variable coefficients]. Ufimskii matematicheskii zhurnal - Ufa Mathematical Journal, Vol. 9, No. 2, 94-103 [in Russian]. 
16 Khubiev, K.U. (2018). Zadachi so smeshcheniem dlia nahruzhennoho uravneniia hiperbolo-parabolicheskoho tipa s operatorom drobnoi diffuzii [Tasks with an offset for a loaded equation of a hyperbaloparabolic type with a fractional diffusion operator]. Vestnik Udmurtskoho universiteta. Seriia Matematika. Mekhsnika. Kompiuternye nauki. - Bulletin of Udmurt University. Math. Mechanic. Computer Sciences, Vol. 28, No. 1, 82-90 [in Russian].

17 Dzhenaliev, M.T. (1995). K teorii lineinykh kraevykh zadach dlia nahruzhennykh differentsialnykh uravnenii [On the theory of linear boundary value problems for loaded differential equations]. Almaty: Komputernyi tsentr TIPM [in Russian].

18 Dzhenaliev, M.T. \& Ramazanov, M.I. (2010). Nahruzhennye uravneniia kak vozmushcheniia differentsialnykh uravnenii [Loaded equations as perturbations of differential equations]. Almaty: Hylym [in Russian].

19 Ogorodnikov, E.N. (2003). Nekotorye kharakteristicheskie zadachi dlia sistem nahruzhennykh differentsialnykh uravnenii i ikh sviaz s nelokalnymi kraevymi zadachami [Some characteristic problems for systems of loaded differential equations and their relations with nonlocal boundary value problems]. Vestnik Samarskoho hosudarstvennoho universiteta. Seriia phys.-math. nauk - Bulletin Samara State Tech. University. Series phys.-math. sciences, Vol. 19, 22-28 [in Russian].

20 Borok, V.M. (1986). O sushchestvovanii i edinstvennosti resheniia zadachi Koshi dlia nahruzhennykh uravnenii [On existence and uniqueness of the solution to the Cauchy problem for loaded equations]. Izvestie vuzov. Seriia Matematika - Izv. universities. Math., No. 4, 8-13 [in Russian].

21 Borok, V.M. (1985). O edinstvennosti resheniia zadachi Koshi dlia sistem lineinykh nahruzhennykh uravnenii [On the uniqueness of the solution to the Cauchy problem for systems of linear loaded equations]. Ukrainskii matematicheskii zhurnal - Ukrainian Mathematical Journal, Vol. 37, No. 1, 108, 109 [in Russian].

22 Dzhenaliev, M.T. \& Ramazanov, M.I. (2004). O zadache Koshi dlia sushchestvenno nahruzhennoho parabolicheskoho uravneniia [On the Cauchy problem for a substantially loaded parabolic equation]. Matematicheskii zhurnal - Mathematical Journal, Vol. 4, No. 3(13), 22-26 [in Russian].

23 Dzhenaliev, M.T. \& Ramazanov, M.I. (2005). O hranichno-nachalnoi zadache dlia sushchestvenno nahruzhennoho parabolicheskoho uravneniia [On boundary - initial problem for a substantially loaded parabolic equation]. Izvestiia NAS RK, Seriia Phys. math., No. 5, 36-43 [in Russian].

24 Lomov, I.S. (1983). Nahruzhennye differentsialnye operatory: skhodimost spektralnykh razlozhenii [Loaded Differential Operators: Convergence of Spectral Expansions]. Differentsialnye uravneniia - Differential Equations, Vol. 19, No. 1, 86-94 [in Russian].

25 Attayev, A.Kh. (2016). Zadacha Hursa dlia nahruzhennoho vyrozhdaiushchehosia hiperbolicheskoho uravneniia vtoroho poriadka s operatorom Hellerstedta v hlavnoi chasti [The Goursat problem for a loaded degenerate second-order hyperbolic equation with the Gellerstedt operator in the principal part]. Vestnik Samarskoho hosudarstvennoho tekhnicheskoho universiteta. Seriia phys.-mat. nauk, No. 1(20), 7-22 [in Russian].

26 Attayev, A.Kh. (2014). Zadacha Hursa dlia nahruzhennoho hiperbolicheskoho uravneniia [Goursat problem for a loaded hyperbolic equation]. Reports of the Adygskaya (Circassian) International Academy of Sciences, Vol. 16, No. 3, 9-12 [in Russian].

27 Attayev, A.Kh. (2013). Zadacha s dannymi na parallelnykh kharakteristikakh dlia nahruzhennoho volnovoho uravneniia [A problem with data on parallel characteristics for a loaded wave equation]. Reports of the Adygha (Circassian) International Academy of Sciences, Vol. 15, No. 2, 25-28 [in Russian].

28 Bitsadze, A.V. (1982). Uravneniia matematicheskoi fiziki [Equations of mathematical physics]. Moscow: Nauka [in Russian]. 